\title{
A Study of the chemical nature of sulfur-containing rocks and Frasch Sulfur in the Al-Mishraq Sulfur field
}

\author{
Hala S. J ${ }^{1}$, Thaeir A. H ${ }^{2}$, Motea O. A ${ }^{3^{*}}$ \\ ${ }^{1,2}$ Department of Chemistry, collage of education for girl, University of Mosul, Mosul, Iraq \\ 3* Mishraq Sulfur State Company, Mosul, Iraq
}

E-mail: ${ }^{1}$ hala.saad@uomosul.edu.iq, ${ }^{2}$ thaer.abd@uomosul.edu.iq, ${ }^{* 3}$ alojmaniojmani@ gmail.com

(Received February 20, 2020; Accepted May 14, 2020; Available online March 01, 2021)

DOI: 10.33899/edusj.2020.126714.1051, (c) 2020, College of Education for Pure Science, University of Mosul.

This is an open access article under the CC BY 4.0 license (http://creativecommons.org/licenses/by/4.0/).

\begin{abstract}
The economic evaluation of the exploration sulfur well depends mainly on the elemental sulfur content and its associationother factors, so a sample of sulfur rocks extracted by a special excavator for one of the exploratory wells was studied and in the form of a bore hole rock. The elemental sulfur ratio reached $12.8 \%$ an important ratio in estimating the explored sulfur reservesand through XRD,the predominant crystalline composition is calcite mineral with hexagonal crystalline form and with a lesser percentage in orthorhombic crystal sulfur this supports one of the theories that explained the formation of sulfur.

Also, the mineMishraqsulfur extracted by the underground melting method (known as the Frasch process) was studied chemically, and the elemental sulfur ratio reached $98.58 \%$ and the bituminous material $1.157 \%$. Also, the SEM scanning electron microscope showed the presence of nanotubes of size (20-44 $\mathrm{nm}$ ) belonging to the carb-sulfur compounds in the SEM, which are observed for the first timein this form.
\end{abstract}

Keyword: Mishraq sulfur, bore hole, frasch

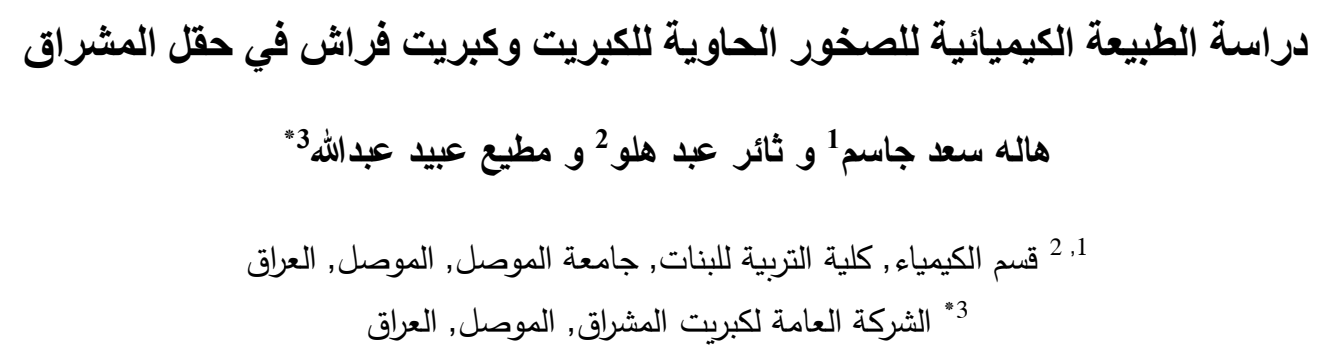

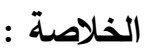

يعتمدالتقييم الاقتصادي للبئر الكبريت المستكثف على نسبة الكبريت الحربشكل اساسي وربطها بعوامل اخرى ، لذا تمت دراسة نموذج

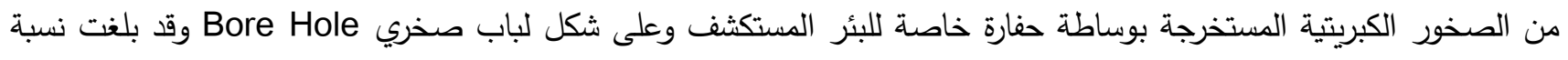
الكبريت العنصري 12.8\% وهذه النسبة مهمة في تقدير احتياطي الكبريت المستكثف، ومن خلال حيود الاشعة السينية XRD كان

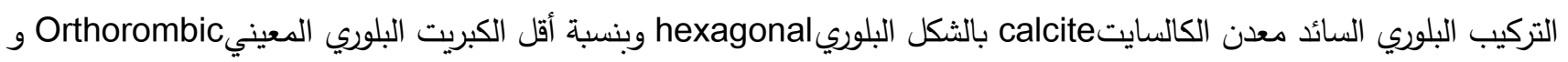
هذا يدعم أحد النظريات التي فسرت تكون الكبريت . 
كما تمت دراسة كبريت المشراق المنجمي المستخرج بطريقة الصهر الجوفي (المعروفة بطريقة فراش Frasch process) كيميائياً وبلغت نسبة الكبريت الحر 98.58\% والمادة القيرية 157 157 ،ومن خلال حيود الأشعة السينية تبين ان الكبريت البلوري المعيني

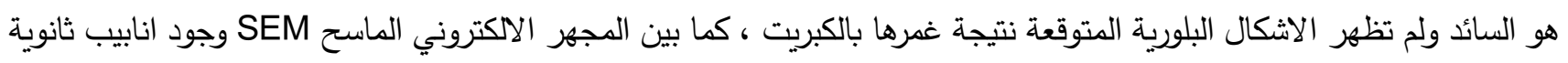
بحجم (20-44nm) تعود للمركبات الكاربوكبريتية في كبريت المشراق المنجمي التي تلاحظ لأول مرة بهذه الهيئة .

الكلمات المفتاحية : كبريت المشراق , اللباب , فراش.

\title{
introduction : المقدمة
}

يتواجد الكبريت العنصري Elemental Sulfur في العراق في خمسة عشر موقعا في تراكيب طيات محدبة كما في المشراق ولزاكة ومكحول وحمرين ونجمة وقصب والمستغل منها حقل كبريت المشراق الذي يقع في شمال العراق على بعد 350 كم شمالبغداد و 45 كم جنوب مدينة الموصل في منطقة تحتوي حقول نفطية وعيون كبريتية ولكون كبريت المشراق من النوع الرسوبي لابد من توافر

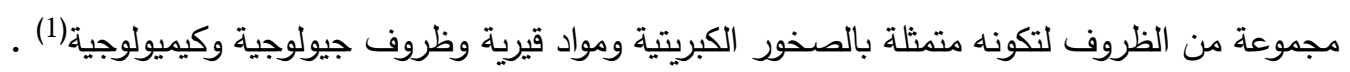

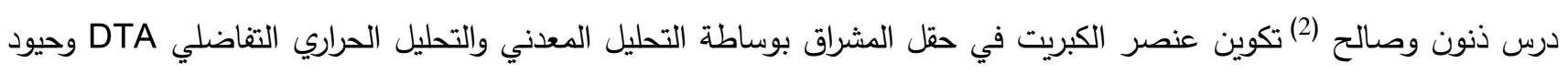

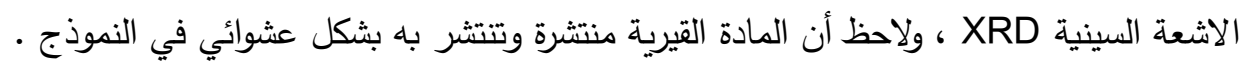

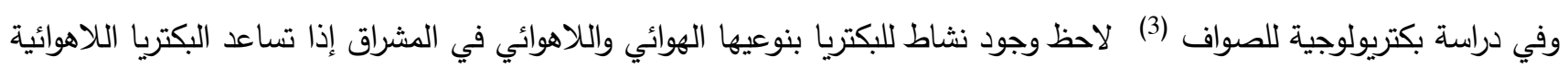
على تكوين الكبريت العنصري Elemental Sulfur فيما تعمل الهوائية على أكسدة الكبريت الى كبريتات وهذا يعلل سبب ورئ وجود كبريت على أعماق سحيق في حقل المشراق • واستنتج الجبوري (4) من خلا نسبة الكبريت الحر في المواد القيرية والمتواجد على عمق يقارب 180m وقبل تعرضها لظروف فراش ونسبته في النفط الخام لحقل القيارة النفطي ان نسبة الكبريت الحر تزداد كلما اتجهنا نحو حقل المشراق. ودرس souaya(5) إضافة الكبريت لإسفلت التبليط ومن خلال دراستهم بطرائق عدة درس التغيرات في التراكيب والمظهر الخارجي

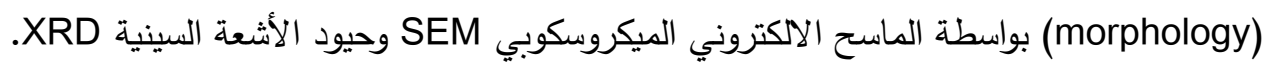
وفي دراستتا الحالية تمت دراسة طبيعة للصخور الحاملة للكبريت وعلى عمق يقارب 200mوالكبريت المنجمي المستخرج وبطرائق كيميائية وطيفية للأول مرة دراسة المظهر الخارجي للكبريت المنجمي بواسطة المجهر الالكتروني الماسح - مطياف تشتت طاقة Scanning electron microscopy - Energy Dispersive X-RY الأشعة السينية

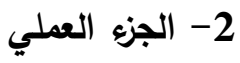 \\ 1-2 \\ Xpert Phillips Holand نوع XRD جهاز حيود الاشعة السينية \\ 2. جهاز المجهر الالكتروني الماسح SEM نوع Tescan Mira3 France \\ 2-2 حساب نسبة الكبريت العنصري في اللباب الصخري(6):
}

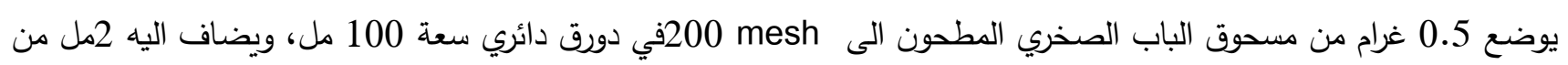
الايثانول ثم 50 مل من المحلول 20\% كبريت دنيت الصوديوم ويربط المكثف العاكس ويصعده لمدة ساعتين مع الرج ويرشحفي قنينة

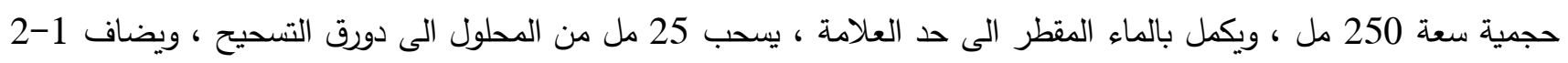

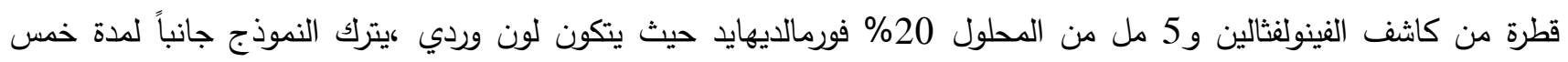


دقائقويضاف حامض الخليك المركز قطرة قطرة حتى اختفاء اللون الوردي ، وسحح المحلول مع محلول 0.1N من اليود وباستعمال محلول النشأ كدليل يسجل حجم اليود وتحسب نسبة الكبريت عنصري :

3-2 تعيين نسبة المادةالقيرية في كبريت المشراق المنجمي(6):

\section{Determination of Bituminous Materials in Raw sulfur}

يوزن في بيكر 50 غم من الكبريتالمنجمي ويضاف إليه 2 مل من الإيثانول (96\%) و 50 مل من محلول 20\% هيدروكسيد

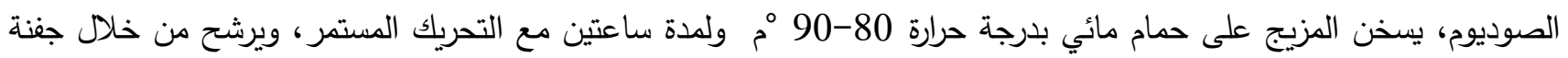

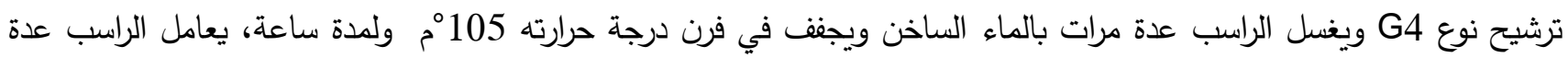

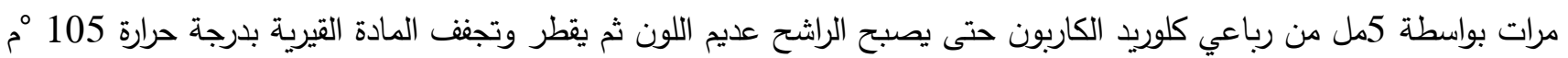

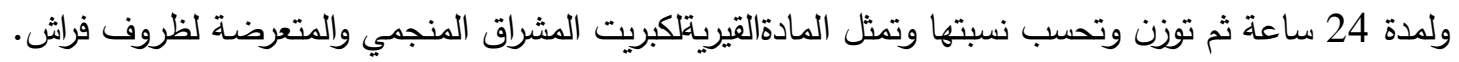

\section{Determination of Carbon and Ash in Raw Sulfur}

4-2 تعيين نسبة الكاربون والرماد في كبريت المشراق(7):

يوضع 5 غرام من الكبريت المنجمي في جفنة خزفية موزونة مسبقاً وتسخن تسخيناً هيناً على مسخن كهربائي لحين انتهاء

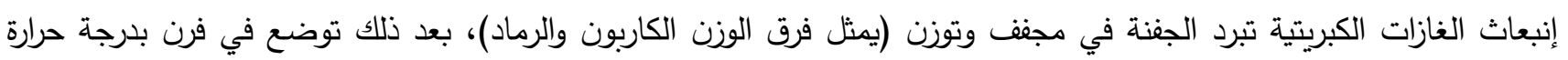
800 م لمدة ساعة، تبرد الجفنة في مجفف ثم توزن (ويمثل فرق الوزن الرماد).... ثم تحسب النسبة المؤية للكاربون والرماد .

5-2 تقدير الحامضية في كبريت المشراق المنجمي( 7):

\section{Determination of Acidity in Raw Sulfur}

يوضع في بيكر 50 غرام من النموذج و 25 مل من الإيثانول و 200 مل ماء مقطر تمزج المكونات جيداً يغطى البيكر بزجاجة ساعة ثم يسخن على مسخن كهربائي حتى الغليان ولمدة 15 دقيقة ييرد الى درجة حرارة الغرفة ثم يرشح في قنينة حجمية

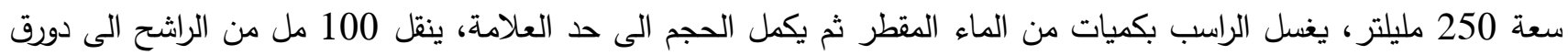

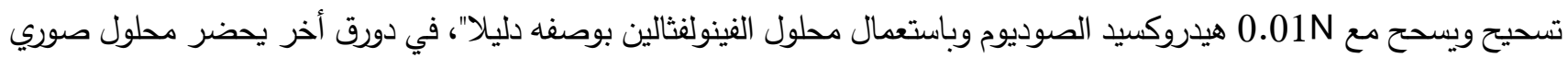

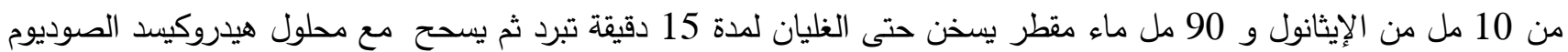
باستعمال الدليل نفسه، ثم تحسب الحامضية على أساس حامض مل مل ملك الكبريتيك

6-2 حساب نسبة الكبريت الكلي والكبريت العضوي والكاربون الحر والكاربون العضوي(4) \%Total sulfur=100- (\%FreeCarbon+ \%Ash + \%Acid +\%Organic)

$\%$ Organic sulfur $=\%$ Total sulfur $-\%$ Free sulfur \%Organic Carbon $=$ \%Total Carbon $-\%$ Free Carbon

$\%$ Free Carbon $=\%$ Total Carbon $-\%$ Bitumen $* 0.82$

7-2 قياس حيود الأثشعة السينية والمجهر الالكتروني الماسح تم قياس حيود الاشعة السينية لمسحوق اللباب الصخري وكبريت المشراق المنجمي بوساطة جهاز حيود الاشعة السينية XRD ،ودراسة المظهر الخارجي لكبريت المشراق المنجمي بواسطة جهاز المجر الاككتروني الماسح SEM الموصوفة في الفقرة 2-1 
Result and discussion : النتائج والمناقشة تعد ترسبات الكبريت العنصري Elemental Sulfur في حقل المشراق الكبريتي إحدى اكبر الترسبات الطباقية ذات الأصل العضوي biogenetic المعروفة في العالم وهي تغطي مساحة ما يقارب 10 كم2 ويتواجد الكبريت في ثلاث طبقات رئيسه (1) .

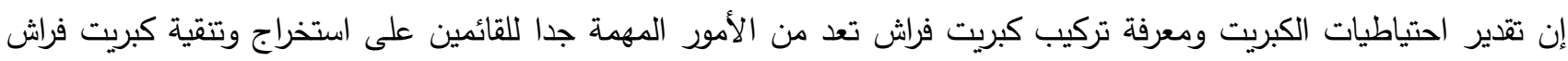
المنجمي ، لذا تم التركيز في دراستنا الحالية على دراسة طبيعة الصخور الحاملة للكبريت لغرض إعطاء صورة مبسطة عن تقدير الاحتياطي ودراسة مفصلة عن طبيعة كبريت المشراق المنجمي بغية التعرف على نسبة المكونات الرئيسة سيما المواد القيرية والتي دري

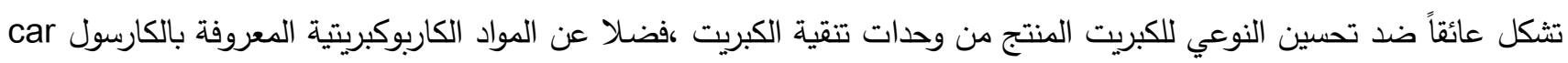
(sul) الكبريتي وتوقفه عن الإنتاج ·

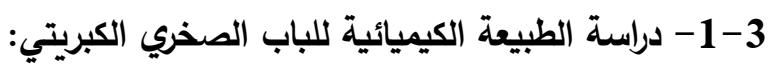
يتم استخراج اللباب الصخري أثناء عملية حفر البئر الكبريتي الإنتاجي او الاستكثافي اما بشكل مراحل متقطعة او مستمرة وينظم

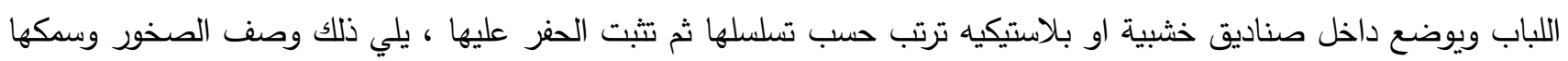

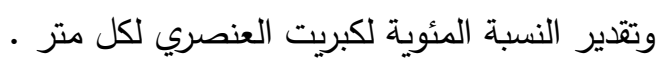
إن تحديد النسبة المئوية للكبريت العنصري في اللباب الصخري (borehole) يعد من الأمور الأساسية والمهمة في تحديد العطاء الكبريتي وتقدير الاحتياطي الكبريتي ولتحقيق ذلك يجب توافر معلومات حقلية ومختبرية تتمثل النسبة المئوية لعمق الطبقة الكبريتية

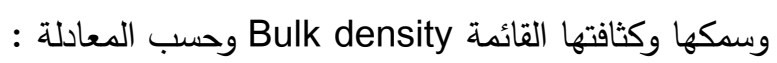
العطاء الكبريتي = معدل \% كبريت العنصري في الطبقة * سمك الطبقة * الكثافة القائمة بلغت نسبة الكبريت العنصري في اللباب الصخري (12.8\%) والتي تدخل ضمن حسابات معدل الكبريت العنصري في الطبقة . ولوحظ ان الصخور الحاملة للكبريت ملوثة بمناطق متفرقة بمواد قيرية والتي تتكون من اسفلتين وبترولين كمواد رئيسه.

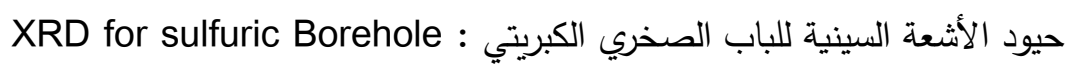
لغرض التعرف على الطبيعة البلورية لمكونات اللباب لصخري الكبريتي ، تم دراسة النموذج بواسطة حيود الاثعة السينية للمسحوق ،

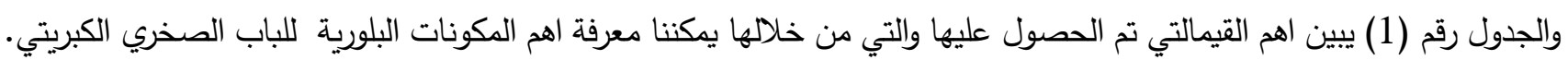

جدول (1): قيم المسافة الذرية d-spacing وضعف زاوية الحيود 20 و معاملات ميلر h k l 1 والنسبة المئوية للثدة النسبية Dن Intensity

\begin{tabular}{|c|c|c|c|c|}
\hline item & $2 \theta$ & d-spacing & Intensity & h kl \\
\hline 1 & 15.559 & 5.6907 & 11.4 & 022 \\
\hline 2 & 23.246 & 3.8233 & 66.4 & 012 \\
\hline 3 & 25.964 & 3.4288 & 26.4 & 026 \\
\hline 4 & 27.889 & 3.1964 & 32.6 & 040 \\
\hline 5 & 26.889 & 3.3130 & 16.6 & 311 \\
\hline 6 & 27.889 & 3.1964 & 32.6 & 040 \\
\hline 7 & 28.853 & 3.0918 & 15.2 & 135 \\
\hline 8 & 29.600 & 3.0154 & 100.0 & 104 \\
\hline 9 & 36.216 & 2.4783 & 58.6 & 110 \\
\hline 10 & 39.588 & 2.2746 & 18.4 & 113 \\
\hline 11 & 43.4160 & 2.0825 & 55.0 & 202 \\
\hline 12 & 47.696 & 1.9051 & 26.4 & 024 \\
\hline 13 & 48.699 & 1.8682 & 35.8 & 116 \\
\hline 14 & 56.839 & 1.6185 & 12.8 & 359 \\
\hline 15 & 60.858 & 1.5087 & 17.4 & 208 \\
\hline
\end{tabular}


نلحظ من الجدول أعلاه وبالاعتماد على الادبيات المعتمدة ومن خلال قيم $2 \theta$ وd-spacing ومستويات ميلر ان الطور البلوري السائد للباب الصخري الكبريتي هو الكلسيات Calcite وبشكل بلوري hexagonal من خلال قيم $2 \theta$ ومستويات ميلر hon والتي تقابلها

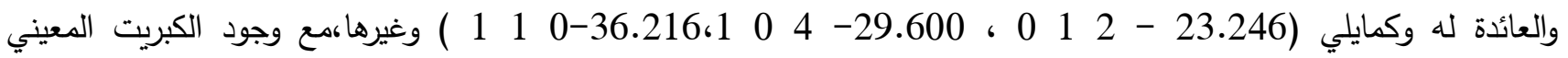
بنسبة اقل من خلال قيم $2 \theta$ ومستويات ميلر hkl العائدة له وكمايلي(orthorhombic)

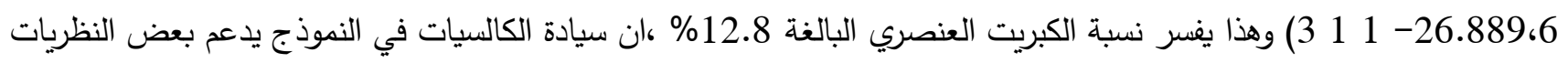

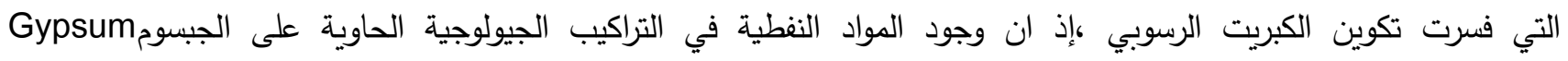
(CaSO $\mathrm{CaSO}_{4}$ (anhydride) $\left.\mathrm{CH}_{2} \mathrm{O}\right)$ في التفاعل العام(8).

$$
\begin{aligned}
\mathrm{CaSO}_{4}+\mathrm{RH} \Longrightarrow \mathrm{S}+\mathrm{CaCO}_{3}+\mathrm{H}_{2} \mathrm{O} \\
\text { والثكل (1) يوضح حيود الاشعة السينية للباب الصخري }
\end{aligned}
$$

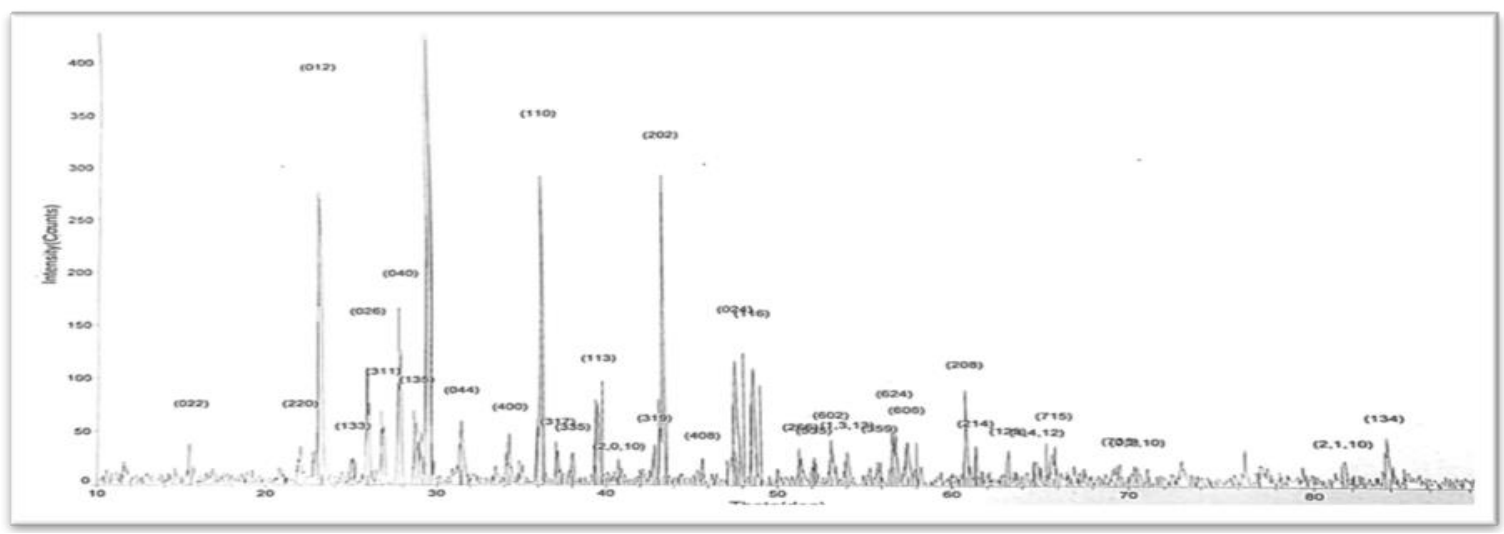

الثكل(1) نمط حيود الاشعة السينية للباب الصخري الكبريتي

3-2-3 دراسة تركيب كبريت فراش المنجمي في منجم المشراق يـ

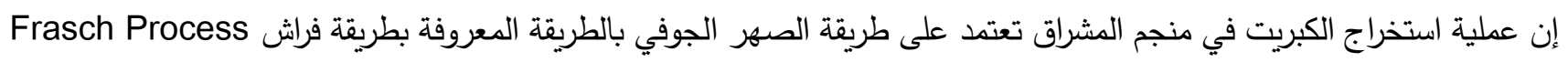

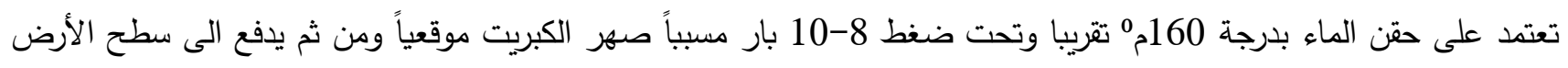
بوساطة الهواء المضغوط. على الرغم من ارتفاع نسبة الكبريت العنصري في كبريت فراش المنجمي التي تصل الى اكثر من 98.5\% في اغلب الأحيان الا أن معرفة نسبة المواد العضوية والمواد الكاربوكبريتية تعد من اهم الأمور الواجب معرفتها قبل دخول الكبريت المنجمي الى وحدات تنقية الكبريت. تم تقدير المكونات الرئيسة لكبريت فراش المنجمي في منجم المشراق وتم ربطها بحسابات معتمدة (4) لغرض إعطاء صورة مفصلة عن

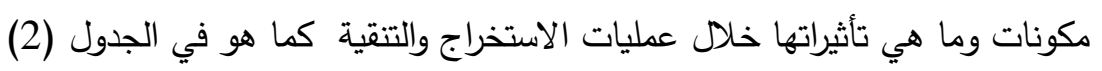




\begin{tabular}{|c|c|}
\hline \% الككون & wt. $\%$ \\
\hline Total Sulfur & 98.71 \\
\hline Elemental Sulfur & $98.58^{*}$ \\
\hline OrganicSulfur & $0.130 *$ \\
\hline Total Carbon & $1.060 *$ \\
\hline Free Carbon & 0.111 \\
\hline Organic Carbon & 1.157 \\
\hline Ash & 0.022 \\
\hline Acidity $\mathrm{H}_{2} \mathrm{SO}_{4}$ & 0.000 \\
\hline
\end{tabular}

يتضح من الجدول (2) ارتفاع في نسبة الكبريت العنصري رغم ذلك فإن للثوائب الموجودة فيه تأثير كبير على استخراجه وتتقيته والمتمثلة بالمواد العضوية الاسفلتية والكاربوكبريتية إذ تثكل هذه المواد عائقاً في وجه التحسين النوعي لكبريت المصفى الناتج عن وحدات تتقية الكبريت ،كما شكلت هذه المواد سبباً رئيساً في تلكؤ إنتاج بعض الآبار الكبريتية واحتمالات توقفها نهائياً نتيجة ما يعرف ولئه بتصلب البئر الكبريتي والذي يحصل اما بتصلب الكبريت السائل نتيجة انخفاض في درجة الحرارة الطبقة الكبريتية المنتجة ، او انسداد البئر بالمواد الاسفلتية والمواد الكاربوكبريتية وإذا ما تمت المعالجات وبعدة إجراءات وبشكل سريع سيؤدي ذلك الى توقف البئر الإنتاجي نهائياً . نان. مما سبق أعلاه نلحظ أهمية إجراءات التحاليل المطلوبة لمعرفة مكونات الكبريت المنجمي سيما المواد العضوية والكاربوكبريتية ، ومن اجل إعطاء صورة أشمل فقد تم دعم هذه النتائج بواسطة تقنية حيود الأثعة السينية XRD والماسح الالكتروني المايكروسكوبي نمط حيود الأثعة السينية لكبريت فراش المنجمي

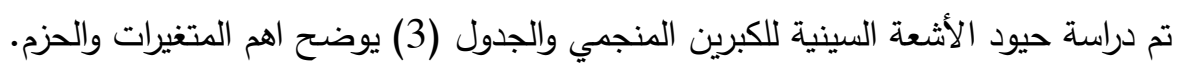

جدول (3) قيم المسافة الذرية d-spacing وضعف زاوية الحيود 02 و معاملات ميلر h kl والنسبة المئوية للثدة النسبية Intensity

\begin{tabular}{|c|c|c|c|c|}
\hline item & $\mathbf{2 \theta}$ & d-spacing & Intensity\% & h kl \\
\hline 1 & 15.695 & 5.6415 & 14.1 & 022 \\
\hline 2 & 23.387 & 3.8006 & 100.00 & 222 \\
\hline 3 & 26.142 & 3.4060 & 24.5 & 026 \\
\hline 4 & 27.006 & 3.2990 & 22.1 & 311 \\
\hline 5 & 27.999 & 3.1841 & 32.1 & 206 \\
\hline 6 & 29.001 & 3.0763 & 26.4 & 135 \\
\hline 7 & 31.695 & 2.8207 & 22.4 & 044 \\
\hline 8 & 37.349 & 2.4057 & 12.2 & 404 \\
\hline 9 & 43.048 & 2.0995 & 24.5 & 2210 \\
\hline 10 & 57.561 & 1.5999 & 19.6 & 606 \\
\hline
\end{tabular}


يظهر من خلال قيم 20و d-spacing ومستويات ميلر للنموذج ان الثكل البلوري السائد هو الكبريت المعيني orthorhombic

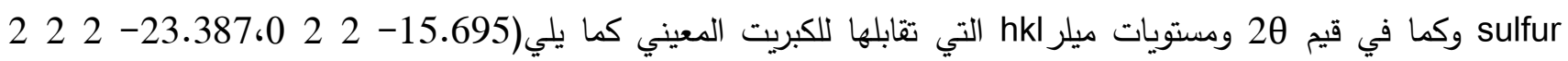

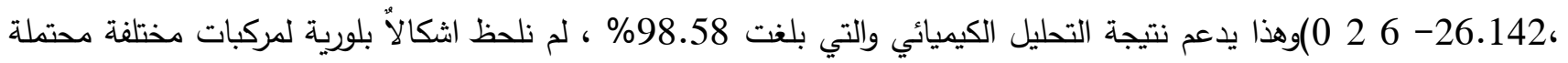
الوجود كالجبسوم والانهيدريد والكلسياتا لدولومايت واطيان المارل Marl clays والتي تتأثر بظروف الاستخراج بسبب احاطتها التامة

بواسطة الكبريت المنصهر . كما في الثكل (2)

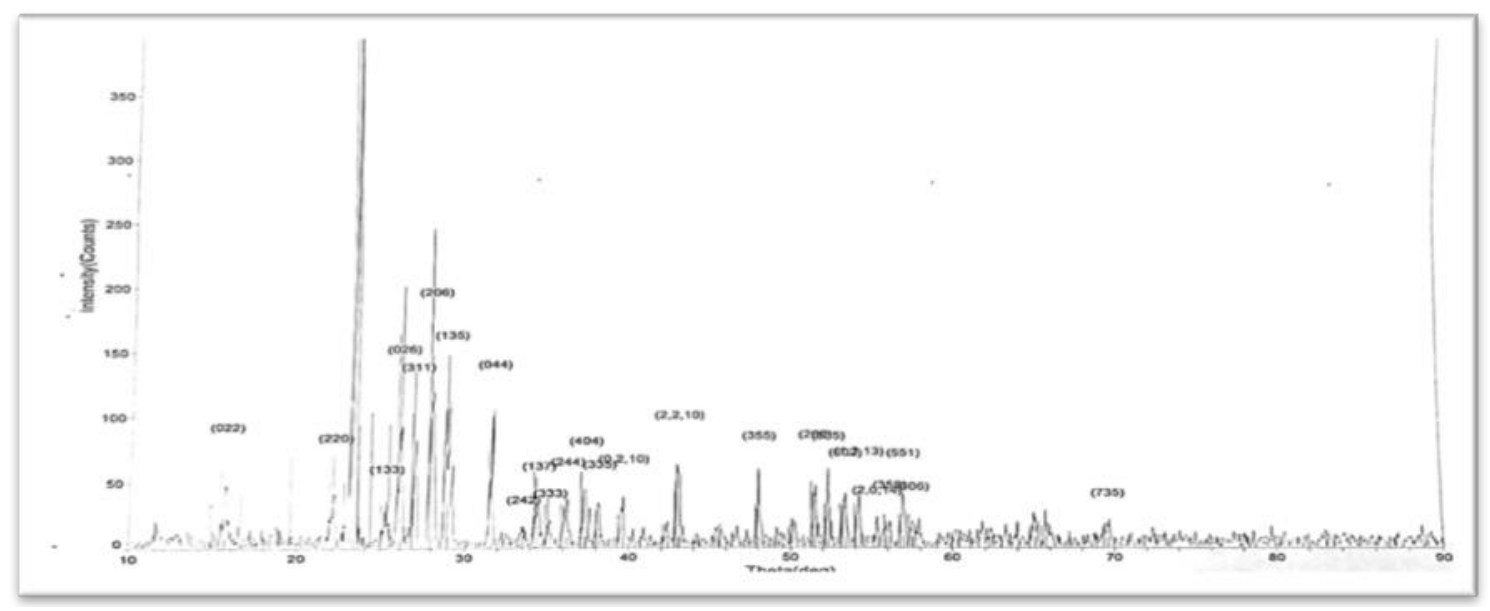

\section{الثكل( 2) نمط حيود الاشعة السينية لكبريت المشراق المنجمي}

شكل مجموع الثوائب في النموذج التي بلغت 1.389\% معضلة حقيقية في استخراج وتتقية الكبريت لذا نلحظ تتوع طرائق تتقية الكبريت المنجمي إذا استخدمت طرائق كيميائية وحرارية وفيزيائية تهدف بمجموعها للحصول على الكبريت نقي يفي بالغرض المنتج Scanning له ، ولغرض استكمال الصورة حول تركيب الكبريت المنجمي تمت دراسة بواسطة تقنية المجهر الأكتروني الماسيح Electron Microscopy

\section{1- تقنية المجهر الاككتروني الماسح لكبريت المشراق المنجمي}

حسب المعلومات المتوافرة من الادبيات تعد دراستنا الأولى للتحليل كبريت المشراق المنجمي بواسطة (SEM) تظهر الصور المكبرة

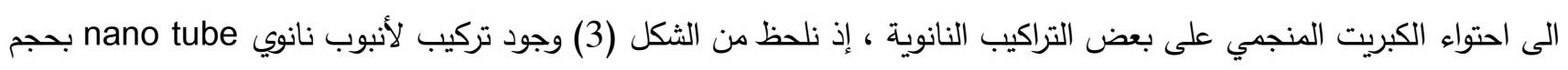
(20.34nm) نعتقد ان هذه التراكيب تعود للمركبات الكاربو كبريتية وتعرف بالكارسول غير الناضج (92.55nm) للكبريت والكرافينوraphene

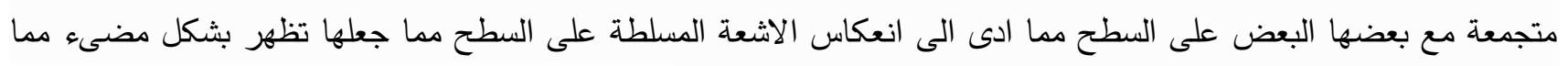

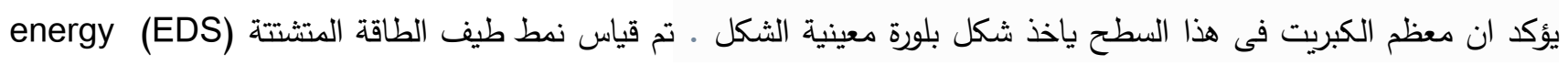

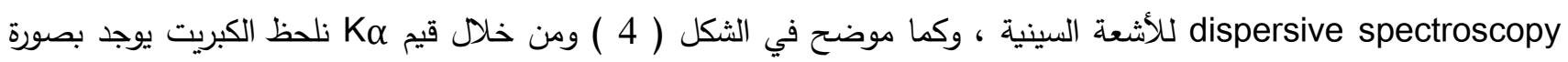
رئيسية ويمثل 84.84\% ثم الكاربون 1\% والاوكسجين 0.15\% ان هذه القيم تدعم تفسيرنا لعدم ظهور أي تراكيب بلورية متوقعة

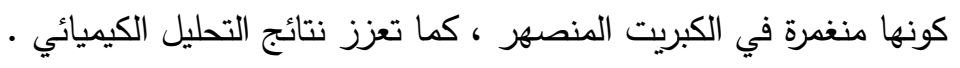




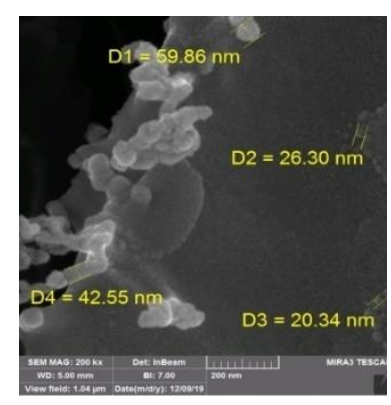

$2 \mu \mathrm{m}$

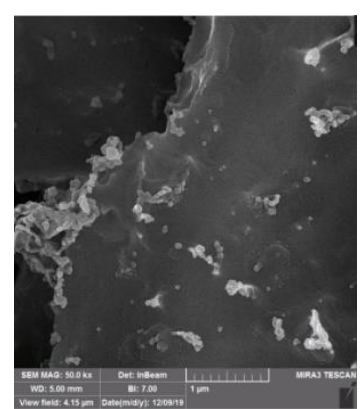

$1 \mu \mathrm{m}$

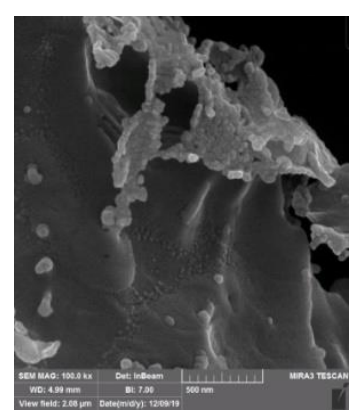

$500 \mathrm{~nm}$

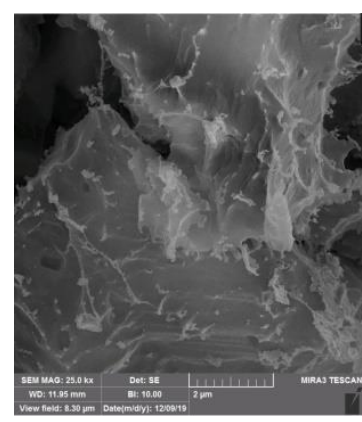

$200 \mathrm{~nm}$

الشكل(3) تحليل المجهز الاككتروني الماسح SEM لكبريت المشراق المنجمي

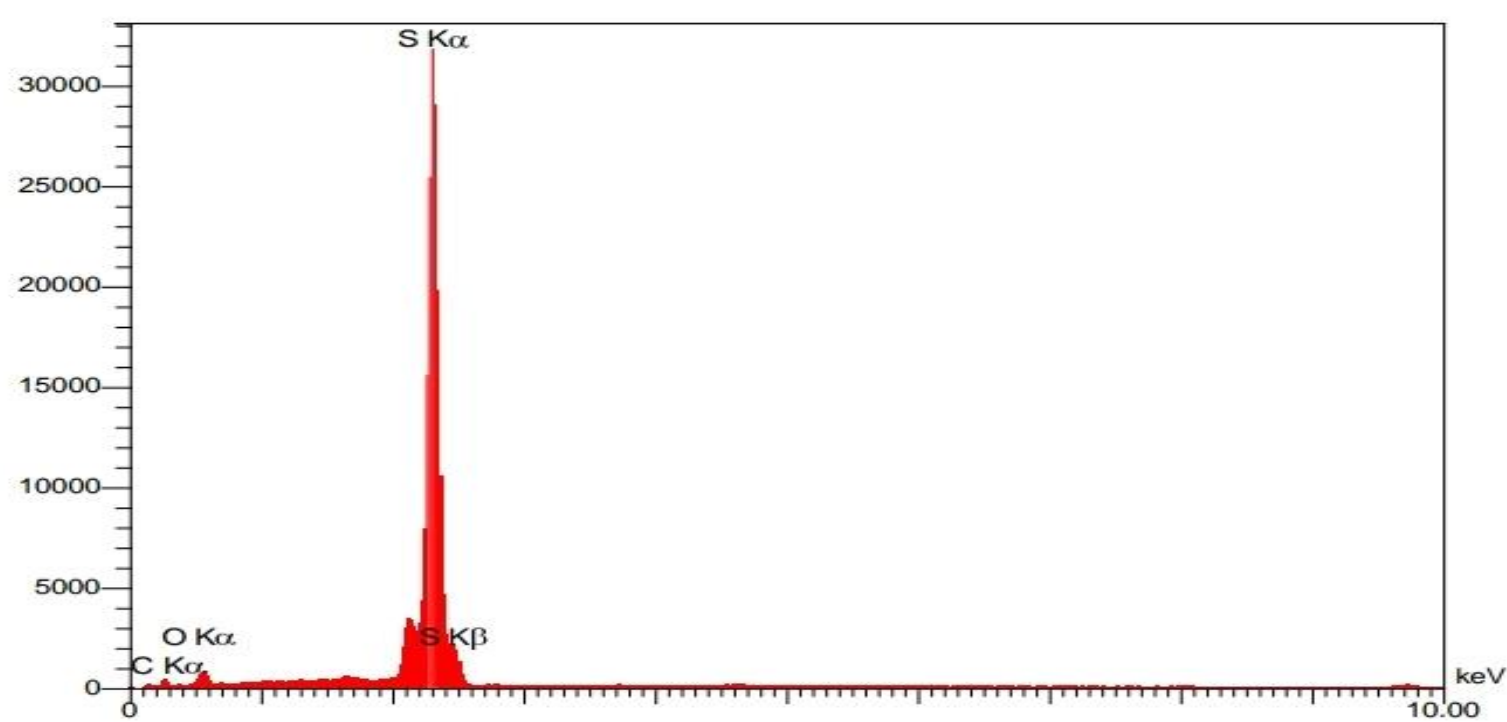

الثكل(4) طيف طاقة تشتت الاشعة السينية eds لكبريت المشراق المنجمي

Conclusion الاستنتاجات

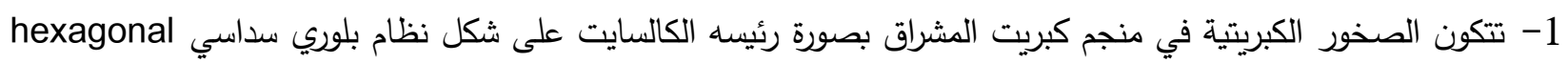
وبنسبة اقل الكبريت العنصري المعيني orthorhombic وتتواجد بأماكن متفرقة مواد نفطية ثقيلة ، ومن خلال هذه المعطيات بكني

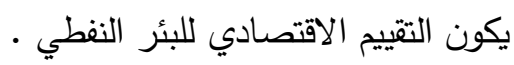

2- يسود الكبريت المعيني orthorhombic بصورة رئيسة في كبريت المشراق المنجمي مع وجود مواد قيرية ثقيلة منتشرة فيه فضلاً عن المركبات الكاربوكبريتية(الكارسول carsul) غير الناضح ، ومن خلال هذه المعطيات يمكن اقتراح الطريقة الملائمة للتنقية.

\section{: Acknowledgement شكر وتقدير}

يتقدم الباحثون بالثكر والتقدير لقسم الكيمياء في كلية التربية للبنات - جامعة الموصل و الثركة العامة لكبريت المشراق وهيأة البحث والتطوير الصناعي في وزارة الصناعة والمعادن لتقديم التسهيلات اللازمة لاكمال هذا البحث ـ 


\section{References المصادر}

1. Baker, J.M.; Cochran, D.E. and Semrad, R. (1979). "Economic geology of the Mishraq active sulfur deposit, northern Iraq”. Economic geology. Vol. 74, No. 2, pp. 484-495

2. Dhannon, H.Y. and Salah, S.A. (1977) :Rafidian J. of Sc. 1, 3 .2

3. Alsawaf F.D.S * Sulfate Reduction and sulfur Deposition in the lows Fars Formation, Northern Iraq Economic Geology, 72,608-618 (1977).

4. Al-Jburi, M.U. "A study of Sulfur Waste obtained from Raw sulfur Purification by Thermal Method"M.sc. thesis University of Mosul(1999).(In Arabic)

5. E.R. Souaya, S.A. Elkholy, A.M.M. Abd El-Rahman , M. El-Shafie,I.M. Ibrahim, Z.L. Abo-Shana (Partial substitution of asphalt pavement withmodified sulfur) Egyptian Journal of Petroleum 24, 483491. (2015)

6. Al-Jburi, M.O.“ A guide of quality control in Mishraq sulfur state company “ 2004, p 7-14

7. William, N. T..“The Sulphur Data Book” Free Port Sulphur Company. P.4(1954).

8. Latif H. Ali “ A Fundamental and Application in Industrial Chemistry “ University of Mosul .(1990)

9.Hyne, J.B. “Don’t produce carsul” Hydrocarbon processing p: 241-244(1982).

10. WookAhna, Kwan g-Bum Kima, Kyu-Nam Jungb, Kyoung-HeeShinb, Chang-Soo Jin "Synthesis and electrochemical properties of a sulfur-multi walled carbonnanotubes composite as a cathode material for lithium sulfur batteries"Journal of Power Sources 202 (2012) 394- 399. 